\title{
NUEVAS ADICIONES PARA LA FLORA DE VERACRUZ
}

\author{
Jaime Ernesto Rivera-Hernández ${ }^{1,4}$, Miguel de Jesús Cházaro-Basañez ${ }^{2}$, Abel \\ Felipe Vargas-Rueda ${ }^{1}$, Feliza Ramón-Farías ${ }^{3}$, Héctor Oliva-Rivera ${ }^{3}$ y Graciela \\ Alcántara-SAlinas ${ }^{1,3}$ \\ ${ }^{1}$ Centro de Estudios Geográficos, Biológicos y Comunitarios, S.C., \\ calle Santa María 13, Unidad Habitacional San Román, \\ 94542 Córdoba, Veracruz, México. \\ ${ }^{2}$ Universidad Veracruzana, Facultad de Biología, Xalapa, Veracruz, México. \\ ${ }^{3}$ Universidad Veracruzana, Facultad de Ciencias Biológicas y Agropecuarias, \\ Campus Peñuela, Córdoba, Veracruz, México. \\ ${ }^{4}$ Autor para la correspondencia: jriverah@geobicom.org
}

\section{RESUMEN}

Se presentan 17 nuevos registros pertenecientes a 11 familias y 13 géneros de angiospermas para los municipios de Acultzingo y Maltrata, en el estado de Veracruz, además de dos especies que son redescubiertas para la entidad. Se aportan datos sobre el hábitat, distribución y endemismo de las especies y se confirma que esta zona forma parte de la provincia florística de Tehuacán-Cuicatlán.

Palabras clave: Acultzingo, flora, Maltrata, nuevos registros, Veracruz.

\begin{abstract}
We report 17 new records of angiosperms belonging to 11 families and 13 genera for Acultzingo and Maltrata municipalities in the state of Veracruz, in addition to two more species representing rediscoveries for the state. Furthermore, data on species habitat, distribution, and endemism are also provided. We confirm the inclusion of this area as part of the Tehuacan-Cuicatlan Floristic Province.
\end{abstract}

Key words: Acultzingo, flora, Maltrata, new records, Veracruz. 


\section{INTRODUCCIÓN}

La República Mexicana ha sido ampliamente reconocida por su gran diversidad vegetal, ya que en ella se encuentran representados prácticamente todos los tipos de vegetación registrados para el mundo (Rzedowski, 1978). Entre las diferentes comunidades vegetales, el matorral xerófilo resalta como uno de los de mayor importancia ecológica, al ocupar $40 \%$ de la superficie vegetal de México, pero sobre todo por contener $60 \%$ de las especies endémicas registradas para el país (Rzedowski, 1978, 1991).

En el estado de Veracruz existen únicamente tres zonas semiáridas en donde prospera el matorral xerófilo: a) la Barranca Santiago, municipio de Huayacocotla, al norte de la entidad, con afinidad florística con el Desierto Chihuahuense; b) el Valle de Perote-Alchichica, en el centro del estado, que tiene relaciones florísticas con el altiplano de Puebla-Tlaxcala-Hidalgo y c) la región de Acultzingo-Maltrata, también en el centro, con relaciones fitogeográficas con el Valle de Tehuacán-Cuicatlán en Puebla y Oaxaca. La comarca de Perote-Alchichica ha sido estudiada por Ramos y González (1972), así como por Sandoval (1984), mientras que las otras dos zonas se han mantenido inexploradas (Rivera-Hernández et al., 2014).

Durante el desarrollo del proyecto de investigación doctoral del primer autor, titulado "Flora y Vegetación del Parque Nacional Cañón del Río Blanco, Veracruz, México y la identificación de sus Áreas Prioritarias de Conservación", resaltó la escasa exploración botánica que se ha llevado a cabo en toda esta región, pero en especial en el sector semiárido de Acultzingo-Maltrata, ya que no se encontró ningún estudio sobre su universo vegetal. Esta comarca, incluso ha permanecido en el anonimato, pues en la literatura botánica contemporánea (Gómez-Pompa, 1977; Gómez-Pompa et al., 2010; Castillo-Campos et al., 2011) no se le menciona ni se le considera como parte de los matorrales xerófilos de Veracruz. Por lo anterior, el conocimiento florístico de esta área se limita a algunas colectas aisladas, en diferentes épocas, realizadas por colectores e investigadores, tales como Eizi Matuda, Eugène Bourgeau, Mateo Botteri, Frederick Müller, Henry E. Seaton, Francisco Ventura y Marino Rosas, entre otros (Rivera-Hernández et al., 2014).

\section{MATERIALES Y MÉTODOS}

Los municipios de Acultzingo y Maltrata se localizan en el centro del estado de Veracruz, en la región de las Altas Montañas, en la frontera con el estado de Puebla; 
además, forman parte del Parque Nacional Cañón del Río Blanco, en las coordenadas geográficas extremas $18^{\circ} 55^{\prime} 48^{\prime \prime} \mathrm{N}, 97^{\circ} 20^{\prime} 02^{\prime \prime} \mathrm{W}$ y $18^{\circ} 37^{\prime} 34^{\prime \prime} \mathrm{N}, 97^{\circ} 12^{\prime} 37^{\prime \prime} \mathrm{W}$ (Fig. 1).

La mayor parte de la vegetación está representada por matorral xerófilo (según la clasificación de Rzedowski, 1978), el cual se ubica entre los 1300 y los 2300 m s.n.m., con la presencia de árboles como son Prosopis laevigata (Humb. et Bonpl. ex Willd.) M.C. Johnst., Pistacia mexicana Kunth, Casimiroa edulis La Llave et Lex., Celtis caudata Planch., Acacia farnesiana (L.) Willd., Agonandra obtusifolia Standl. y algunas cactáceas y asparagáceas arborescentes como Stenocereus pruinosus (Otto ex Pfeiff.) Buxb., S. stellatus (Pfeiff.) Riccob., Neobuxbaumia macrocephala (F.A.C. Weber ex K. Schum.) E.Y. Dawson y Nolina parviflora (Kunth) Hemsl. Entre los arbustos presentes en el área destacan Gochnatia obtusata S.F. Blake, Rhus standleyi F.A. Barkley, Zanthoxylum fagara (L.) Sarg., Z. limoncello Planch. et Oerst., Bouvardia longiflora (Cav.) Kunth, B. castilloi Borhidi et García Gonz., Condalia mexicana Schltdl., Galphimia speciosa C.E. Anderson, Desmodium orbiculare Schltdl., Quer-

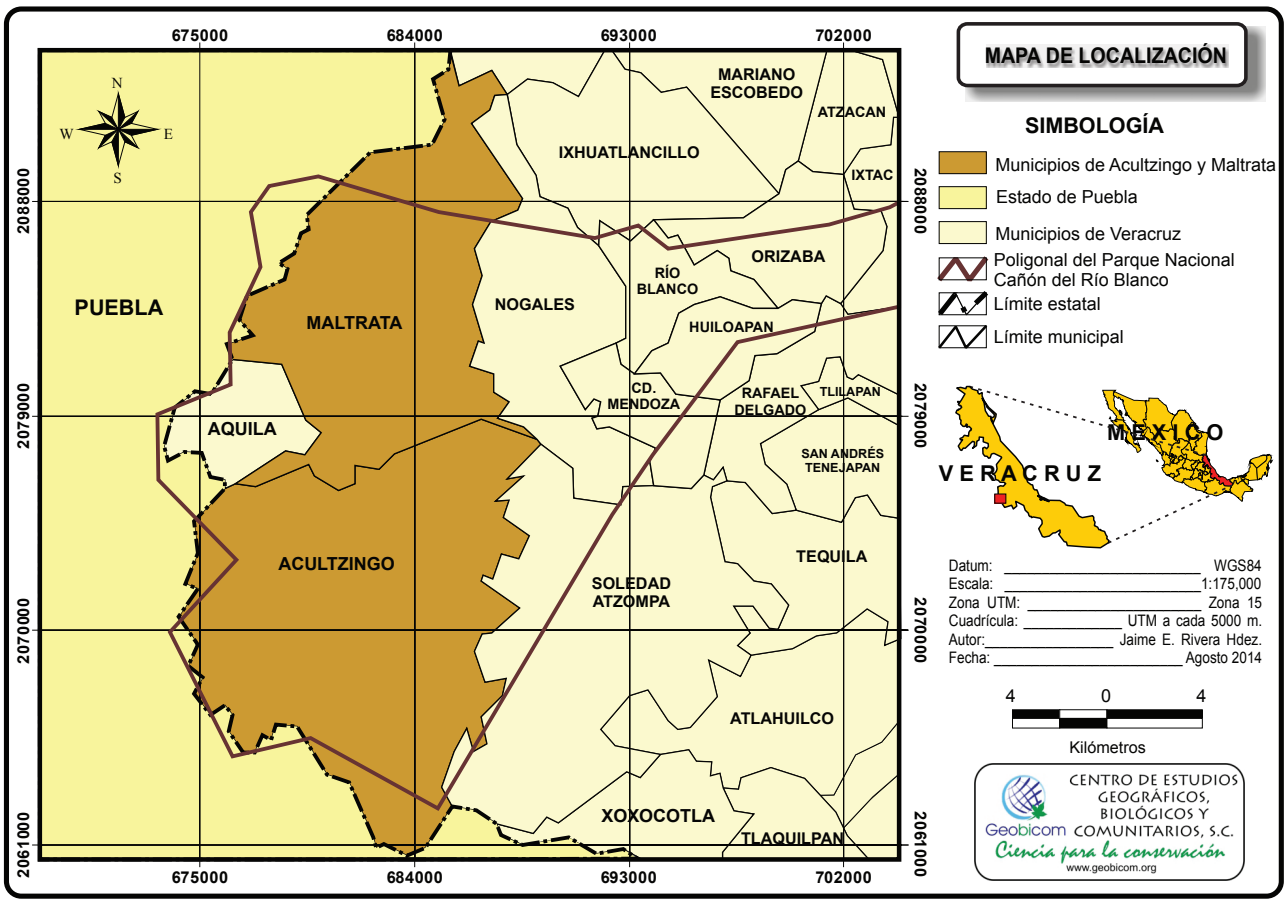

Fig. 1. Mapa de localización del área de estudio modificado de Instituto Nacional de Estadística y Geografía (Anónimo, 2010). 
cus sebifera Trel. y Q. mexicana Bonpl. Los elementos herbáceos son abundantes, especialmente en temporada de lluvias; entre ellos se pueden mencionar a Mirabilis viscosa Cav., Oxalis corniculata L., O. decaphylla Kunth, Tillandsia spp., Echinopepon pubescens (Cogn.) Rose, Mentzelia hispida Willd., Eucnide hirta (Pav. ex G. Don) H.J. Thomps. et W.R. Ernst, Sedum spp., Echeveria rubromarginata Rose e Ipomoea conzattii Greenm., entre muchos otros. También es posible encontrar diversas plantas arrosetadas terrestres, tales como Agave angustifolia Haw. y Hechtia bracteata Mez, además de cactáceas como Coryphantha pycnacantha (Mart.) Lem., Ferocactus robustus (Karw. ex Pfeiff.) Britton et Rose, Mammillaria haageana subsp. acultzingensis (Linzen et al.) R.D. Hunt, M. polyedra Mart., Opuntia parviclada S. Arias et Gamma, O. pilifera F.A.C. Weber, O. pubescens J.C. Wendl. ex Pfeiff., O. tomentosa Salm-Dyck y $O$. aff. lasiacantha Pfeiff. (Rivera-Hernández et al., 2014).

En las partes más altas de ambos municipios, también existe el bosque de Quercus (encino) (según la clasificación de Rzedowski, 1978), entre los 2300-2700 m s.n.m., dominando en el estrato arbóreo Quercus rugosa Née, Q. glabrescens Benth., Q. laurina Bonpl., Q. castanea Née, Pinus patula Schltdl. et Cham., Arbutus xalapensis Kunth, Prunus serotina subsp. capuli (Cav.) McVaugh, Crataegus mexicana DC., C. gracilior J.B. Phipps, Lippia myriocephala Schltdl. et Cham., Alnus acuminata Kunth, Oreopanax echinops (Schltdl. et Cham.) Decne. et Planch., Cornus excelsa Kunth, Salix paradoxa Kunth, Buddleja parviflora Kunth y B. cordata Kunth, además de algunos otros. Entre los arbustos destacan Ribes ciliatum Humb. et Bonpl. ex Roem. et Schult., Ceanothus caeruleus Lag., Tournefortia hirsutissima L., Cestrum fasciculatum (Schltdl.) Miers, Calliandra houstoniana (Mill.) Standl., Monnina xalapensis Kunth, Bouvardia ternifolia (Cav.) Schltdl., Cercocarpus macrophyllus C.K. Schneid. y C. fothergilloides Kunth. Las plantas herbáceas son un componente importante del bosque; entre las que podemos destacar están Cuphea aequipetala Cav., Conopholis alpina Liebm., Lamourouxia rhinanthifolia Kunth, Govenia liliacea (Lex.) Lindl., Escobedia grandiflora (L.f.) Kuntze, Heuchera orizabensis Hemsl., Spigelia longiflora M. Martens et Galeotti, Pinguicula moranensis Kunth, Echeandia mexicana Cruden y Sprekelia formosissima (L.) Herb., así como varias especies más (Fig. 2).

Entre 2012 y 2014 se realizaron exploraciones botánicas en diferentes localidades de los municipios de Acultzingo y Maltrata. Los ejemplares obtenidos fueron herborizados por los métodos tradicionales (Lot y Chiang, 1986) y se depositaron en el herbario “Dr. Jerzy Rzedowski Rotter" de la Facultad de Ciencias Biológicas y Agropecuarias de la Universidad Veracruzana, Zona Orizaba-Córdoba (CORU); se enviaron duplicados al herbario del Instituto de Ecología, A.C. en Xalapa, Veracruz 

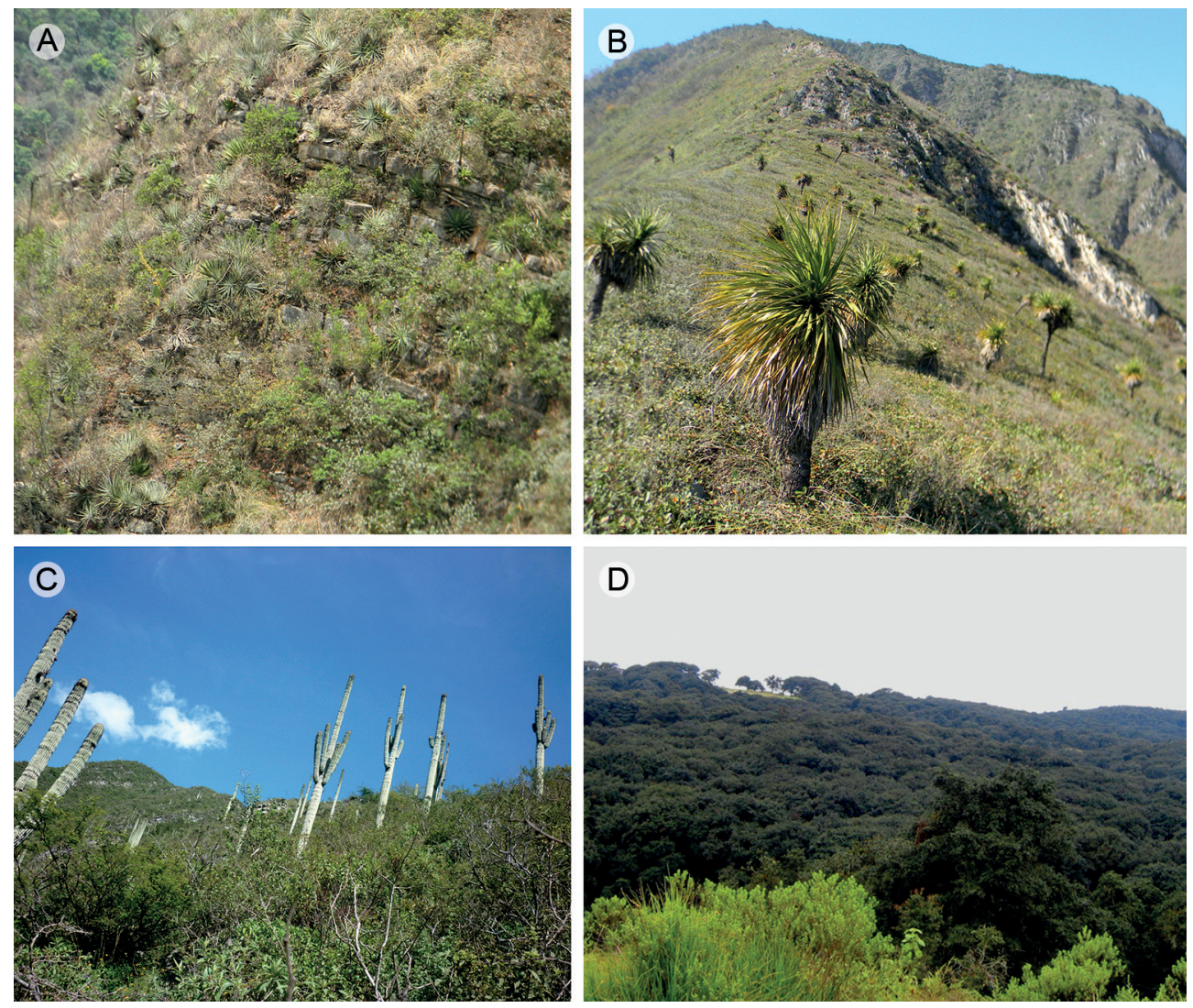

Fig. 2. Diferentes vistas de la vegetación donde prosperan las especies reportadas en este artículo. A. matorral dominado por Hechtia bracteata; B. matorral dominado por Quercus sebifera y Nolina parviflora; C. matorral de Neobuxbaumia macrocephala; D. bosque de encino dominado por Quercus rugosa, Q. laurina y Q. glabrescens. Fotografías de J. E. Rivera-Hernández.

(XAL), así como al Herbario Nacional del Instituto de Biología de la Universidad Nacional Autónoma de México (MEXU). Para la nomenclatura de las familias botánicas, se siguió la propuesta de Angiosperm Phylogeny Group (2009).

\section{RESULTADOS}

Durante estas exploraciones se encontraron 17 especies de plantas que no habían sido previamente reportadas para el estado de Veracruz y dos más que represen- 
tan redescubrimientos para la entidad, ya que se habían registrado anteriormente de colectas muy antiguas y no se habían vuelto a colectar. La mayoría de las especies tratadas en este estudio (17) proceden del matorral xerófilo y únicamente dos del encinar. Solo de estas últimas se especifica en el texto el tipo de vegetación donde habitan.

\section{ARALIACEAE}

Aralia humilis Cav. Árbol que se distribuye desde el suroeste de los Estados Unidos hasta Guatemala (Medina-Lemos, 1994). Sosa (1979) reporta a esta especie para Veracruz, citando únicamente el ejemplar de Müller 1426 (GH) y otros dos existentes en el herbario K, sin aportar más datos. Posteriormente, López-Ferrari (1993) la menciona para el estado, y Medina-Lemos (1994) la registra en el Valle de Tehuacán-Cuicatlán, sin incluirla para Veracruz. En este estudio se considera a esta especie como un redescubrimiento, pues las colectas aquí citadas representan las primeras después de la de Müller, del siglo XIX. La especie se encontró en los municipios de Acultzingo y Maltrata, en donde es un árbol poco común (Figs. 3C y 4). Especímenes examinados: México, Veracruz, Mpio. Acultzingo, cerro Xochío, paraje La Organera, $1 \mathrm{~km}$ al NO del poblado Sierra de Agua, alt. 1720 m, 1845'34" N, 97¹4'54" W, 21 diciembre 2012, J. E. Rivera H. et al. 5318 (CORU, XAL); cerro Xochío, paraje La Organera, 1 $\mathrm{km}$ al NO del poblado Sierra de Agua, alt. 1590 m, 1845'33" N, 97¹4'37" W, 15 mayo 2013, A. F. Vargas R. et al. 475 (CORU, MEXU, XAL); cerro Xochío, paraje La Organera, $1 \mathrm{~km}$ al NO del poblado Sierra de Agua, alt. 1720 m, 1845'34" N, 97'14'54" W, 1 marzo 2014, G. Torres C. et al. 1438 (CORU, MEXU, XAL). Mpio. Maltrata, Maltrata, $1 \mathrm{~km}$ en línea recta al S, por el camino a Zacatonal, alt. $1740 \mathrm{~m}, 18^{\circ} 47^{\prime} 56^{\prime \prime}$ N, 97¹6'52" W, 7 junio 2014, A. F. Vargas R. et al. 1204 (CORU, MEXU, XAL).

\section{ASPARAGACEAE}

Agave ghiesbreghtii Lem. ex Jacobi. Presente en Guatemala y México, donde se le ha reportado de los estados de Chiapas, Guerrero, Oaxaca y Puebla (García-Mendoza, 2011); ahora registrada en los municipios de Acultzingo y Maltrata, en donde es un elemento común (Fig. 4). Especímenes examinados: México, Veracruz, Mpio. Acultzingo, cerro Xochío, paraje La Organera, a $1 \mathrm{~km}$ al NO del poblado Sierra de Agua, alt. 1720 m, 1845'34" N, 97¹4'54" W, 15 mayo 2013, J. E. Rivera H. et al. 5743 (CORU, MEXU, XAL). Mpio. Maltrata, cerro al SO de Maltrata a $1.5 \mathrm{~km}$ en línea recta del centro de Maltrata, alt. 2100 m, 1847'31" N, 97¹7'36" W, 17 mayo 2014, A. F. Vargas R. et al. 1171 (CORU, MEXU, XAL). 
Rivera-Hernández et al.: Adiciones a la flora de Veracruz
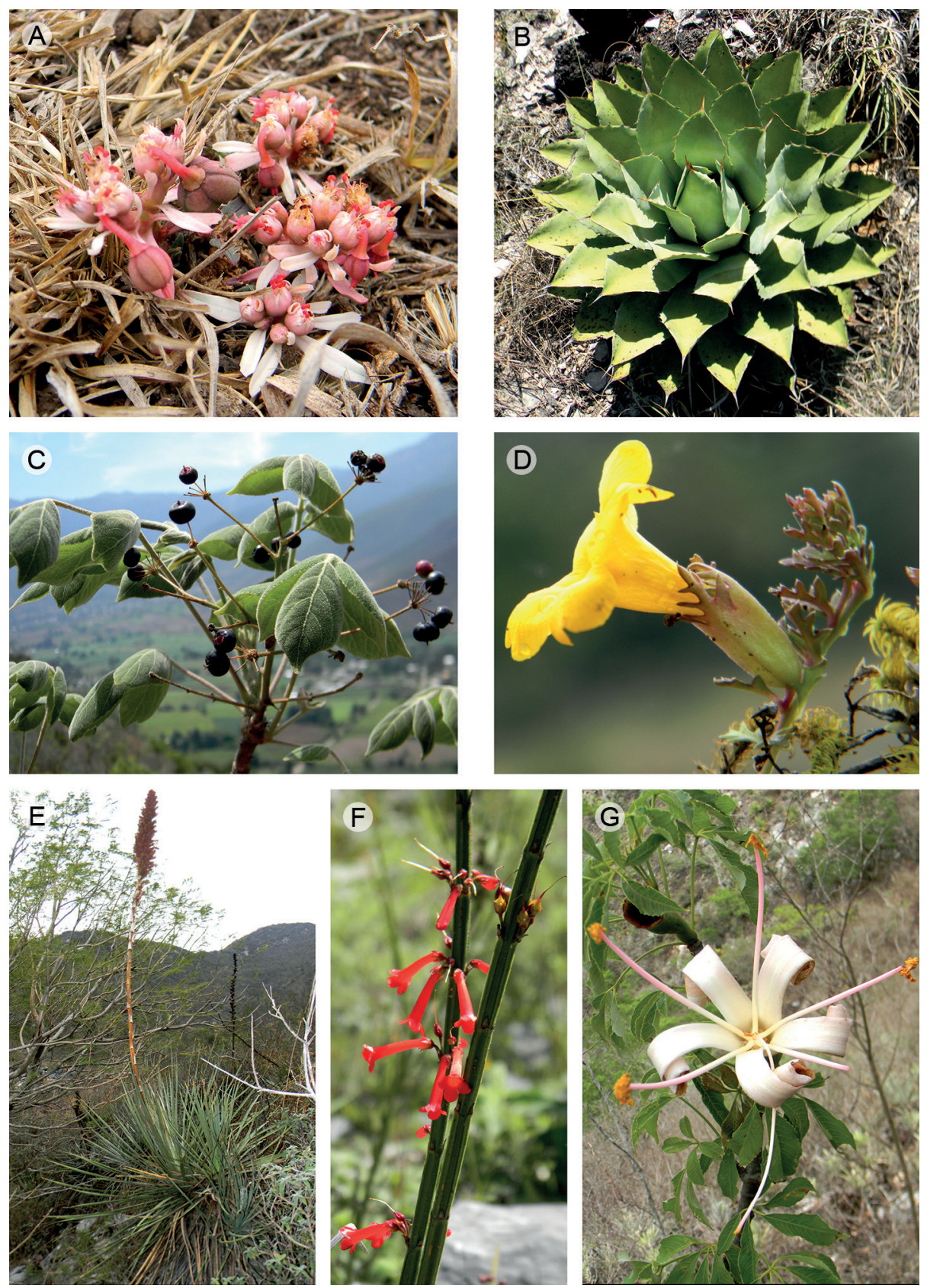

Fig. 3. A. Euphorbia radians, B. Agave potatorum, C. Aralia humilis, D. Silvia prostrata, E. Dasylirion serratifolium, F. Russelia obtusata, G. Ceiba aesculifolia subsp. parvifolia. Fotografías de J. E. Rivera-Hernández. 


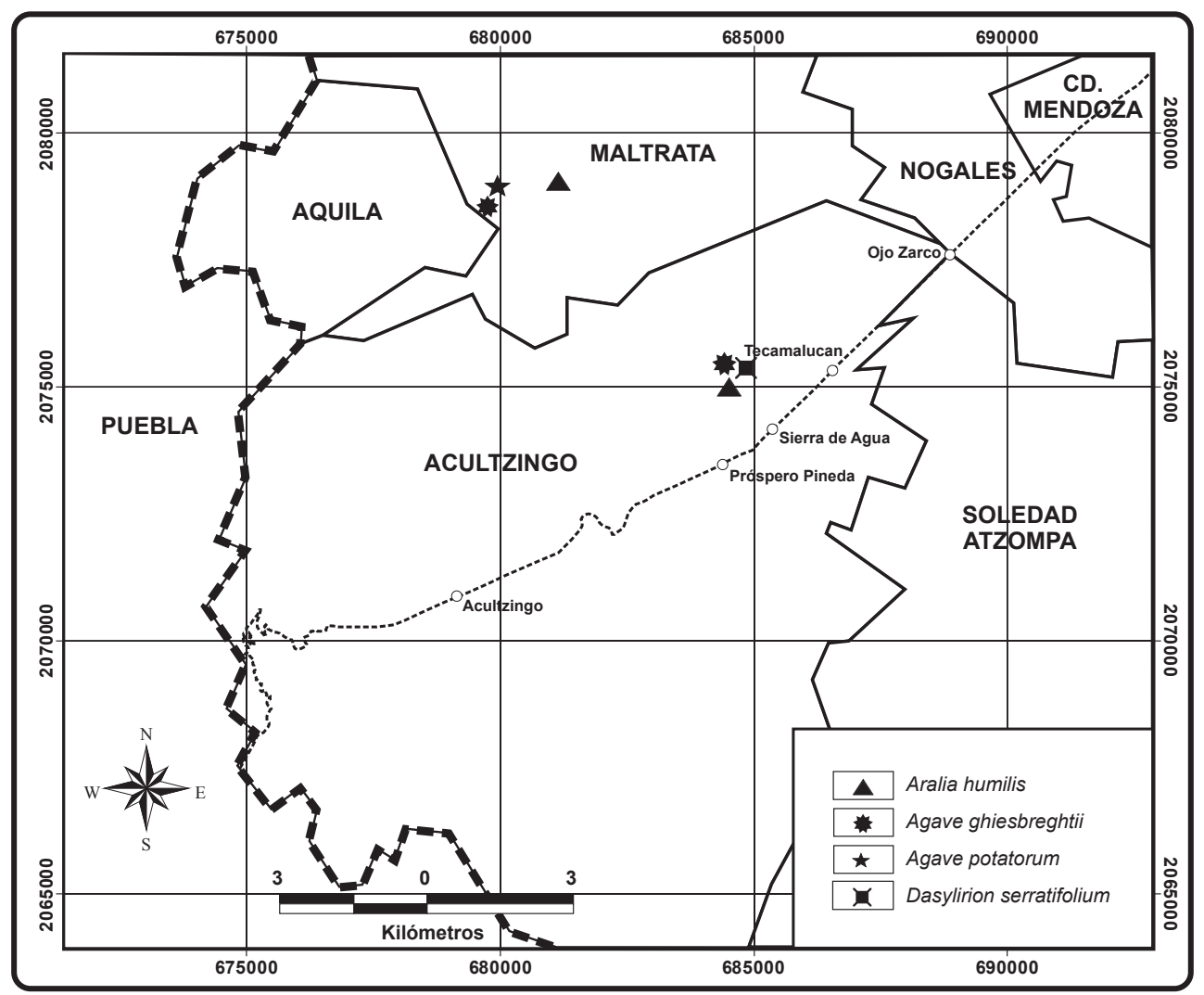

Fig. 4. Mapa de distribución en el área de estudio de Aralia humilis, Agave ghiesbreghtii, A. potatorum y Dasylirion serratifolium.

Agave potatorum Zucc. Especie endémica de México, previamente conocida solo de Oaxaca y Puebla (García-Mendoza, 2011), ahora extendiendo su distribución al municipio de Maltrata, donde representa un elemento común (Figs. 3B y 4). Espécimen examinado: México, Veracruz, Mpio. Maltrata, cerro al SO de Maltrata a 1.5 $\mathrm{km}$ en línea recta del centro del poblado, alt. $2100 \mathrm{~m}, 18^{\circ} 47^{\prime} 31^{\prime \prime} \mathrm{N}, 9^{\circ} 1^{\prime} 36^{\prime \prime} \mathrm{W}, 17$ mayo 2014, A. F. Vargas R. et al. 1196 (CORU, MEXU, XAL).

Dasylirion serratifolium (Karw. ex Schult. f.) Zucc. Planta endémica de México, solo reportada del estado de Oaxaca, al sur del Valle de Tehuacán-Cuicatlán (Rivera y Solano, 2012) y ahora registrada en el municipio de Acultzingo, siendo una especie de abundancia regular (Figs. 3E y 4). Especímenes examinados: México, 
Veracruz, Mpio. Acultzingo, cerro Xochío, paraje La Organera, a $1 \mathrm{~km}$ al NO del poblado Sierra de Agua, alt. 1720 m, 18 ${ }^{\circ} 45^{\prime} 34^{\prime \prime}$ N, 97¹4'54" W, 18 septiembre 2012, J. E. Rivera H. et al. 4945 (MEXU, XAL); cerro Xochío, paraje La Organera, a $1 \mathrm{~km}$ al NO del poblado Sierra de Agua, alt. $1720 \mathrm{~m}, 18^{\circ} 45^{\prime} 34^{\prime \prime} \mathrm{N}, 97^{\circ} 14^{\prime} 54^{\prime \prime}$ W, 21 de diciembre del 2013, J. E. Rivera H. et al. 5304 (CORU, MEXU).

\section{BURSERACEAE}

Bursera aspleniifolia Brandegee. Considerada una especie endémica de México, conocida solamente de los estados de Oaxaca y Puebla (Medina-Lemos, 2008), ahora registrada también del municipio de Acultzingo (Fig. 5). Planta muy parecida a $B$. copallifera, de la que difiere en el patrón de las nervaduras en el envés de los folíolos, pues $B$. copallifera las presenta muy prominentes, formando los nervios terciarios un retículo bien definido, notándose por el haz una superficie ampuloso-rugosa. A su vez, en B. aspleniifolia, la nervadura central y las secundarias también son conspicuas, pero las terciarias están ausentes y por lo tanto no se forma un retículo, además de que las nervaduras secundarias son paralelas entre sí. Estas especies comparten el hábitat en Acultzingo, al igual que en el Valle de Tehuacán-Cuicatlán. Especímenes examinados: México, Veracruz, Mpio. Acultzingo, cerro Xochío, $1 \mathrm{~km}$ al N del poblado Puente de Guadalupe, Alt. $1420 \mathrm{~m}$, 1845'38.0" N, 97¹4'53.6" W, 27 mayo 2012, M. J. Cházaro B. et al. 10315 (CORU, XAL); cerro Xochío, paraje La Organera, $1 \mathrm{~km}$ al NO del poblado Sierra de Agua, alt. $1580 \mathrm{~m}, 18^{\circ} 45^{\prime} 33^{\prime \prime} \mathrm{N}, 9^{\circ} 14^{\prime} 37^{\prime \prime} \mathrm{W}, 15$ mayo 2013, A. F. Vargas R. et al. M. 471 (CORU, MEXU, XAL).

Bursera copallifera (DC.) Bullock. Especie endémica de México, reportada para Colima, Guerrero, Jalisco, México, Michoacán, Morelos, Nayarit, Puebla, Oaxaca y Zacatecas (Medina-Lemos, 2008). Ahora se le registra del municipio de Acultzingo, en elevaciones entre 1400 y $1600 \mathrm{~m}$, conviviendo con otras especies de Bursera (Fig. 5). Espécimen examinado: México, Veracruz, Mpio. Acultzingo, cerro Xochío, paraje La Organera, alt. 1720 m, 1845'34" N, 97014'54" W, 18 septiembre 2012, J. E. Rivera H. et al. 4930 (CORU, MEXU).

Bursera schlechtendalii Engl. Árbol con una amplia distribución geográfica en México y en Guatemala. En nuestro país se distribuye en Coahuila, Tamaulipas, San Luis Potosí, Zacatecas, Querétaro, Hidalgo, Jalisco, Morelos, Puebla, Guerrero, Oaxaca, Chiapas, Yucatán y Campeche (Rzedowski y Guevara-Fefer, 1992). Es im- 


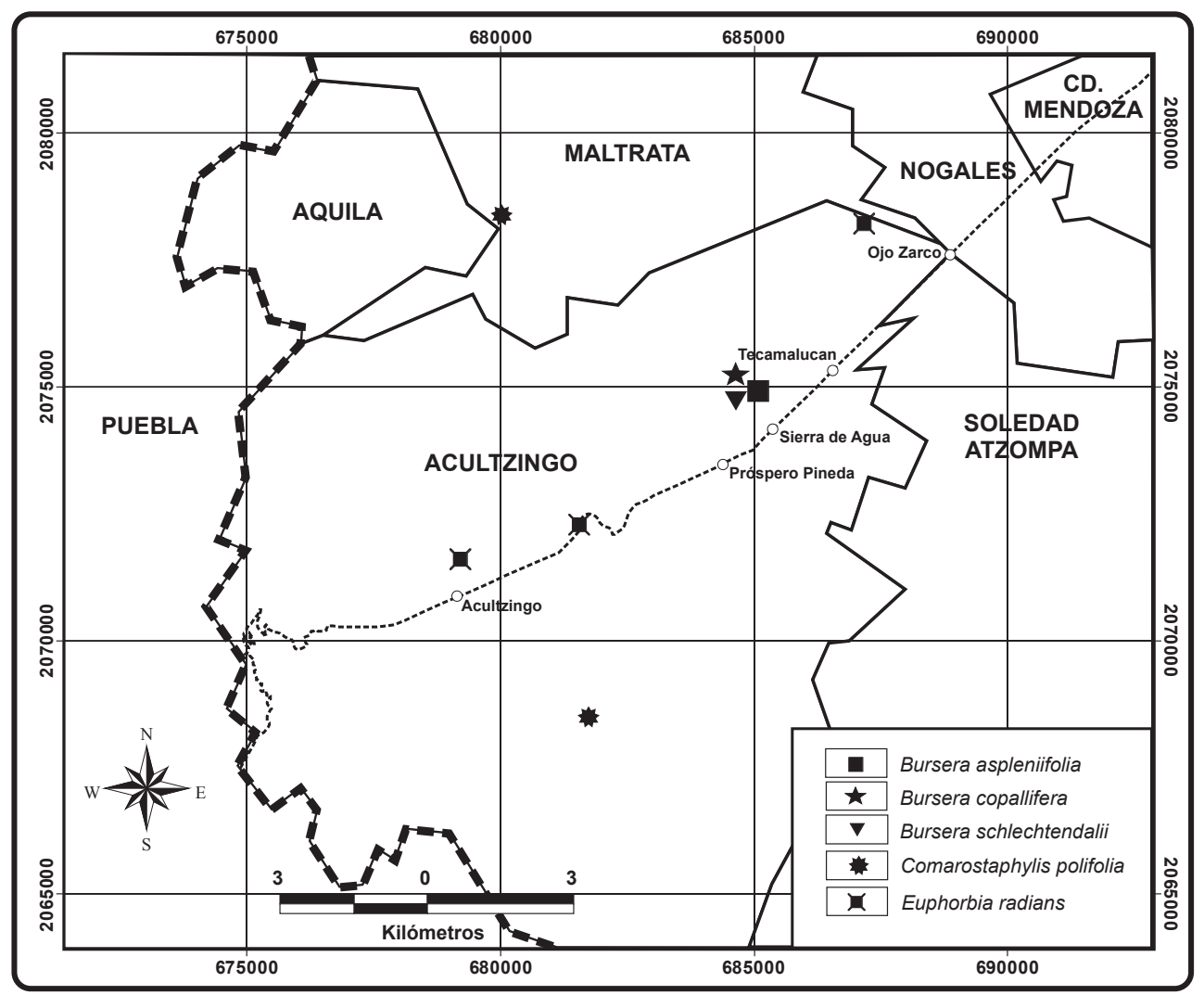

Fig. 5. Mapa de distribución en el área de estudio de Bursera aspleniifolia, B. copallifera, B. schlechtendalii, Comarostaphylis polifolia y Euphorbia radians.

portante resaltar que Rzedowski y Guevara-Fefer (1992) mencionan a esta especie de Veracruz, sin embargo, Rzedowski y Calderón (1996) no la incluyen como parte de la flora del estado, indicando que se reportó previamente, pero con base en identificaciones incorrectas. Por esta razón y porque no se encontró ningún otro ejemplar de herbario de Bursera schlechtendalii para Veracruz, aquí se le considera como un nuevo registro para el estado, del municipio de Acultzingo, en donde su abundancia es regular (Fig. 5). Esta planta se diferencia de las otras de Bursera presentes en el área de estudio por tener hojas unifolioladas y corteza exfoliante rojiza (Fig. 6G). Especímenes examinados: México, Veracruz, Mpio. Acultzingo, cerro Xochío, $1 \mathrm{~km}$ al N del poblado Puente de Guadalupe, Alt. 1470 m, 18²5'22.4" N, 97²14'40.1" W, 27 mayo 2012, M. J. Cházaro B. et al. 10317 (CORU, XAL); cerro Xochío, en el paraje 
La Organera, alt. $1720 \mathrm{~m}, 18^{\circ} 45^{\prime} 34^{\prime \prime} \mathrm{N}, 97^{\circ} 14^{\prime} 54^{\prime \prime} \mathrm{W}, 21$ diciembre 2012, J. E. Rivera H. et al. 5310 (MEXU); La Organera, cerro al N de Sierra de Agua, propiedad de Aguaxinola, alt. $1580 \mathrm{~m}, 18^{\circ} 45^{\prime} 33^{\prime \prime} \mathrm{N}, 9^{\circ} 14^{\prime} 37^{\prime \prime} \mathrm{W}, 15$ mayo 2013, J. E. Rivera H. y A. F. Vargas R. 5746 (CORU, MEXU, XAL); cerro Xochío, paraje La Organera, a $1 \mathrm{~km}$ al NO del poblado Sierra de Agua, alt. $1720 \mathrm{~m}, 18^{\circ} 45^{\prime} 34^{\prime \prime} \mathrm{N}, 97^{\circ} 14^{\prime} 54^{\prime \prime} \mathrm{W}$, 18 septiembre 2012, J. E. Rivera H. et al. 4937 (CORU, MEXU); cerro Xochío, paraje la Organera, a $1 \mathrm{~km}$ al NO del poblado Sierra de Agua, alt. $1720 \mathrm{~m}, 18^{\circ} 45^{\prime} 34^{\prime \prime}$ N, 97²1'54" W, 16 mayo 2014, A. F. Vargas R. et al. 1163 (CORU, MEXU, XAL). Además de las tres especies de Bursera aquí descritas, en la región de Acultzingo también prospera B. fagaroides (Kunth) Engl.

\section{ERICACEAE}

Comarostaphylis polifolia (Kunth) Zucc. ex Klotzsch subsp. polifolia. Especie endémica de México, presente desde Coahuila y Chihuahua hasta el norte y oeste de Oaxaca (Diggs, 1995). No se reporta en la bibliografía para el estado; sin embargo, uno de los autores de este artículo ya previamente la había colectado de Huayacocotla, Veracruz (Cházaro y Hernández 3956, MEXU), sin haberla incluido en alguna publicación, por lo que ahora se registra de Acultzingo, Maltrata y Huayacocotla. En Acultzingo y Maltrata es habitante común en las partes medias de los cerros (Figs. 5 y 6E ). Especímenes examinados: México, Veracruz, Mpio. Acultzingo, cerro por el camino a El Potrero, alt. 1890 m, 1842'10" N, 97'16'17" W, 20 diciembre 2012, J. E. Rivera H. et al. 5287 (CORU, XAL). Mpio. Maltrata, cerro al SO de Maltrata, a $1.5 \mathrm{~km}$ en línea recta del centro del poblado, alt. $2100 \mathrm{~m}, 18^{\circ} 47^{\prime} 31^{\prime \prime} \mathrm{N}, 97^{\circ} 17^{\prime} 36^{\prime \prime} \mathrm{W}$, 17 mayo 2014, A. F. Vargas R. et al. 1172 (CORU).

\section{EUPHORBIACEAE}

Euphorbia radians Benth. var. radians. Elemento herbáceo que habita desde el suroeste de los Estados Unidos (sur de Arizona) hasta México (Dressler, 1961; Mostul y Cházaro, 1996), donde se le reporta de Aguascalientes, Coahuila, Chihuahua, Distrito Federal, Durango, Guanajuato, Hidalgo, Jalisco, Estado de México, Michoacán, Oaxaca, Puebla, Querétaro, San Luis Potosí y Sonora; ahora también de la zona de Acultzingo, con abundancia regular en las partes más bajas de los cerros (Fig. 5). Localmente se le conoce con los siguientes nombres comunes: "estrella de tierra", "camote de rey", "colecitas" (Fig. 3A). Esta planta fácilmente pasa desapercibida, debido a su pequeño tamaño y crecimiento pos- 

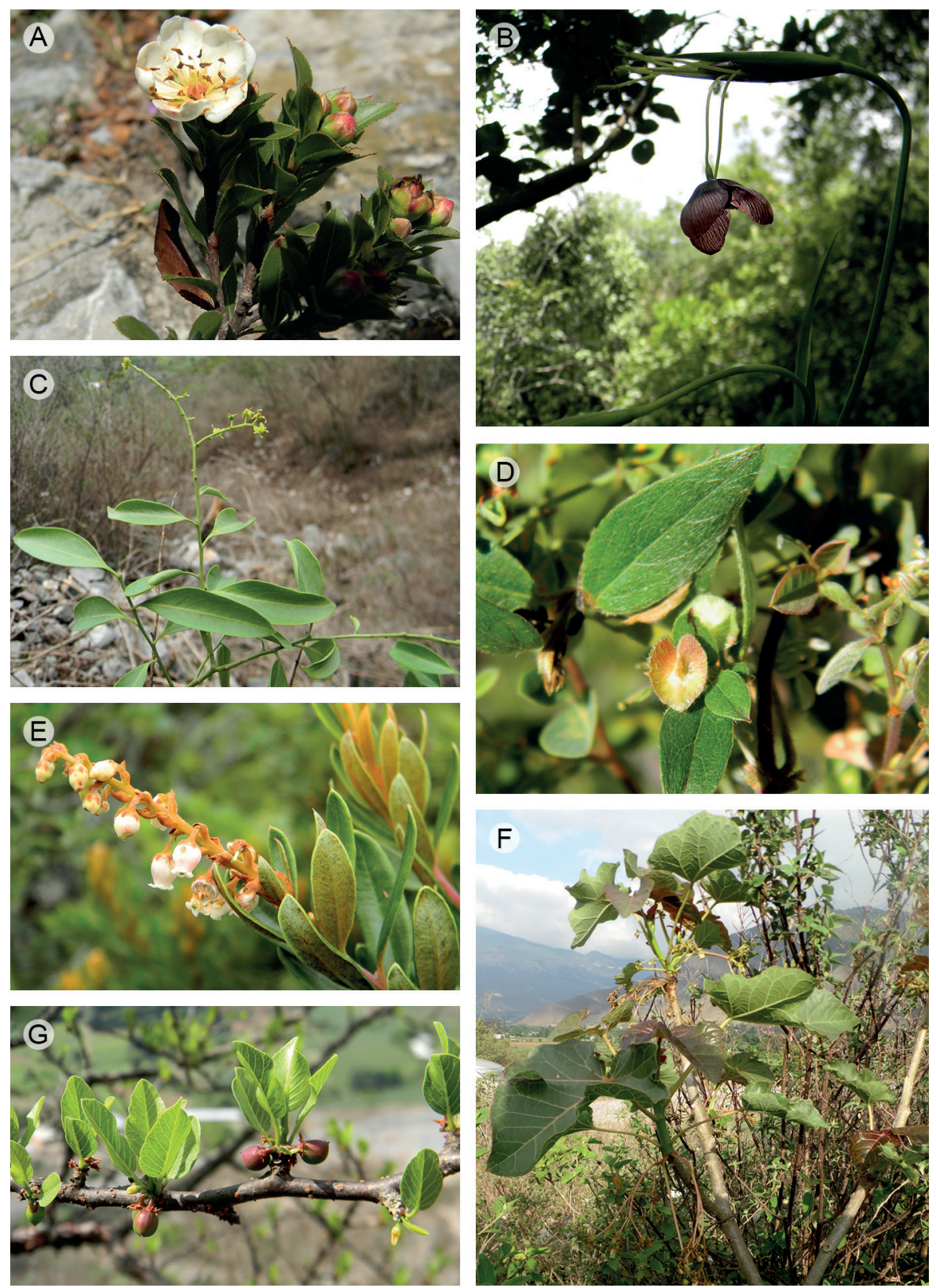

Fig. 6. A. Lindleya mespiloides B. Tigridia galanthoides, C. Agonandra racemosa, D. Gaudichaudia implexa, E. Comarostaphylis polifolia, F. Jatropha ciliata, G. Bursera schlechtendalii. A-E, G, Fotografías de J. E. Rivera-Hernández, F. Fotografia de A. F. VargasRueda. 
trado, siendo evidente únicamente en floración. Rzedowski y Calderón (1987) consideran que existen dos variedades de la especie; la planta colectada en Acultzingo corresponde a Euphorbia radians var. radians Benth., la cual se distingue por las brácteas de la inflorescencia bien desarrolladas y atractivas, el involucro del ciatio blanquecino a rosado, las glándulas del involucro de 1 a 4 (5), las hojas linear-lanceoladas a lineares y porque crece en pastizales y matorrales xerófilos. Especímenes examinados: México, Veracruz, Mpio. Acultzingo, carretera federal Orizaba-Tehuacán, delante de Tecamalucan hacia Acultzingo, cerca de la parada "El Mezquite", alt. 1580 m, 1844'00" N, 97¹6'34" W, 2 marzo 2013, J. E. Rivera H. et al. 5512 (CORU, MEXU, XAL); cerro al N de Acultzingo, por la ermita, alt. $1690 \mathrm{~m}, 18^{\circ} 43^{\prime} 41^{\prime \prime} \mathrm{N}, 9^{\circ} 17^{\prime} 50^{\prime \prime} \mathrm{W}, 2$ marzo 2013, J. E. Rivera H. et al. 5542 (CORU, XAL); $3 \mathrm{~km}$ antes de Acultzingo, sobre la carretera federal Orizaba-Acultzingo, 7 marzo 1992, H. Oliva R. y F. Ramón F. 1072 (CORU, MEXU); $2.5 \mathrm{~km}$ en línea recta al $\mathrm{O}$ de Ojo Zarco, entrando por la desviación a Rancho Viejo, alt. 1460 m, 18²4'20" N, 97'13'27" W, 17 marzo 2014, A. F. Vargas R. 1015 (CORU, MEXU, XAL).

Jatropha ciliata Sessé ex Cerv. Endémica de México, conocida del Distrito Federal, Estado de México, Morelos, Puebla y Oaxaca (Rodríguez et al., 2009; Rzedowski, G. C. de, J. Rzedowski y col., 2001). Ahora también en el municipio de Acultzingo, donde es poco común y restringida a las partes bajas de los cerros (Fig. 7). Esta especie, junto con Euphorbia radians, puede estar en peligro de desaparecer por la extensa agricultura que se practica en las partes bajas de Acultzingo (Fig. 6F). Especímenes examinados: México, Veracruz, Mpio. Acultzingo, cerro Xochío, $1 \mathrm{~km}$ al $\mathrm{N}$ del poblado Puente de Guadalupe, alt. $1450 \mathrm{~m}, 18^{\circ} 45^{\prime} 19.3^{\prime \prime} \mathrm{N}, 9^{\circ} 14^{\prime} 45.8^{\prime \prime} \mathrm{W}, 27$ mayo 2012, M. J. Cházaro B. et al. 10314 (CORU); base del cerro Xochío, $700 \mathrm{~m}$ al NO del poblado Sierra de Agua, antes de cruzar las vías, alt. $1450 \mathrm{~m}, 18^{\circ} 45^{\prime} 18^{\prime \prime} \mathrm{N}, 97^{\circ} 14^{\prime} 46^{\prime \prime}$ W, 16 mayo 2014, A. F. Vargas R. et al. 1168 (CORU, FCME, MEXU, XAL); paraje Peña Roja, 1.2 al O del poblado Próspero Pineda, alt. 1590 m, 1844'42" N, 97¹6'01" W, 21 julio 2014, J. E. Rivera H. et al. 6218 (CORU).

\section{IRIDACEAE}

Tigridia galanthoides Molseed. Planta endémica de México, se le encuentra en los estados de Guerrero, Oaxaca y Veracruz. Reportada por Espejo y López-Ferrari (1998) de Orizaba, a partir de la colecta de H. E. Seaton 525 de 1891 (GH) y no se localizaron más colecciones de herbario. Ahora se registra nuevamente para Veracruz 
de Acultzingo, en bosque de encino, en donde es una especie escasa, representando un redescubrimiento en el estado (Figs. 6B y 7). Espécimen examinado: México, Veracruz, Mpio. Acultzingo, parte alta del cerro por el camino a El Potrero, alt. 2590 m, 1841'26" N, 97²16'47" W, 18 agosto 2013, A. F. Vargas R. et al. 734 (CORU, MEXU).

\section{MALPIGHIACEAE}

Gaudichaudia galeottiana (Nied.) Chodat. Planta endémica de México, solo se conocía los estados de Puebla y Oaxaca (Jessup, 2002), pero ahora se encontró en Acultzingo, en donde es una especie escasa (Fig. 7). Especímenes examinados: Mé-

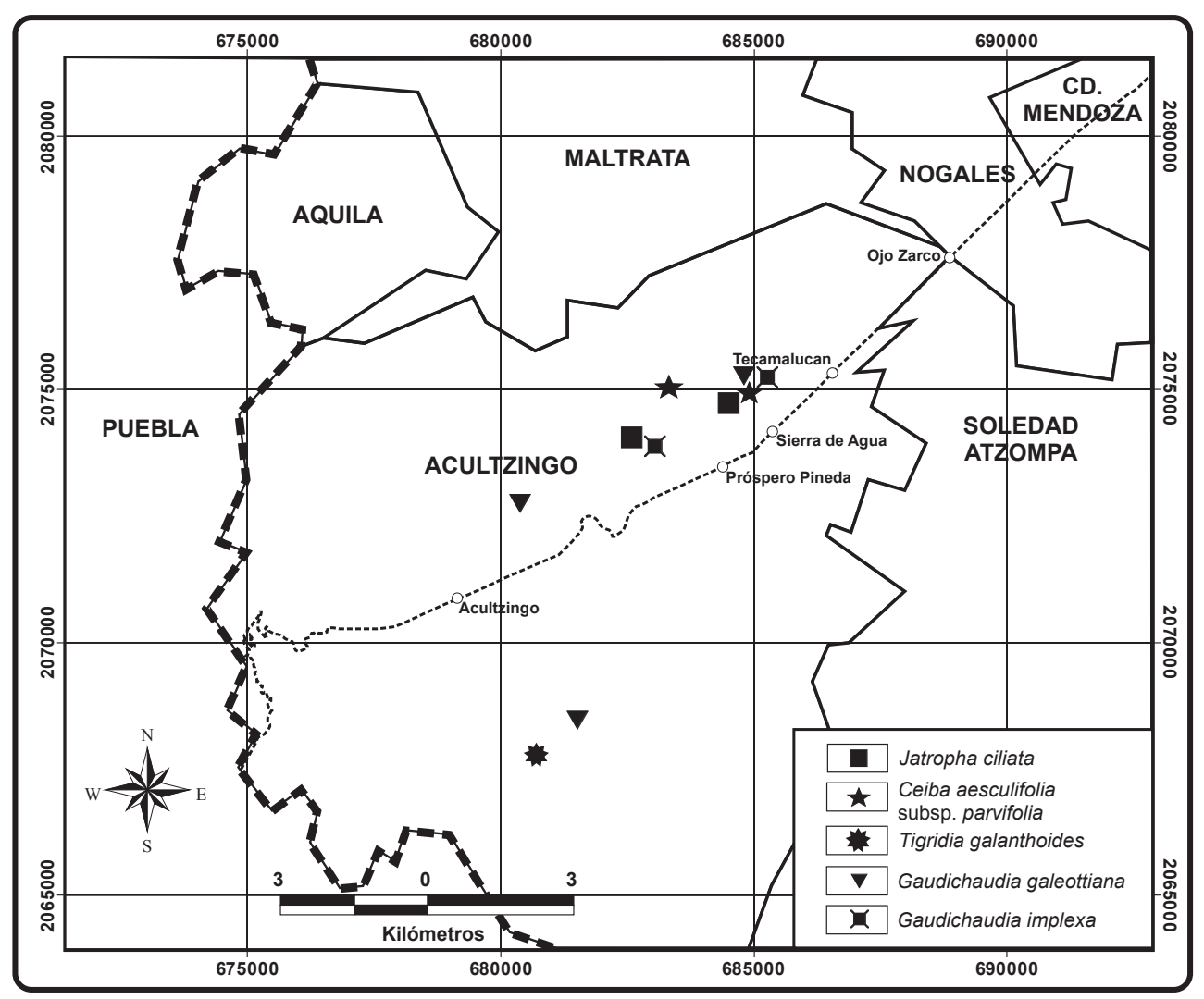

Fig. 7. Mapa de distribución en el área de estudio de Jatropha ciliata, Ceiba aesculifolia subsp. parvifolia, Tigridia galanthoides, Gaudichaudia galeottiana y G. implexa. 
xico, Veracruz, Mpio. Acultzingo, cerro por el camino a El Potrero, alt. $1890 \mathrm{~m}$, $18^{\circ} 42^{\prime} 10^{\prime \prime} \mathrm{N}, 9^{\circ} 16^{\prime} 17^{\prime \prime} \mathrm{W}, 1$ septiembre 2012, J. E. Rivera H. y A. F. Vargas R. 4796 (CORU); Lindavista, $700 \mathrm{~m}$ por el camino de enfrente de la parada "El Mezquite", alt. 1630 m, 18 $44^{\circ} 25^{\prime \prime} \mathrm{N}, 9^{\circ} 17^{\prime} 17^{\prime \prime} \mathrm{W}, 20$ octubre 2012, J. E. Rivera H., et al. 5076 (CORU, XAL); cerro Xochío, paraje La Organera, a $1 \mathrm{~km}$ al N de Sierra de Agua, Propiedad de Aguaxinola, alt. 1590 m, 18² $45^{\prime} 33^{\prime \prime}$ N, 97²1 '37" W, 5 octubre 2013, J. E. Rivera H. et al. 6099 (CORU, MEXU, XAL).

Gaudichaudia implexa S.L. Jessup. Especie endémica de México, pero de amplia distribución en el norte y centro del país, en los estados de Puebla, Jalisco, Nuevo León, Tamaulipas, San Luis Potosí, Querétaro, Guanajuato, Oaxaca, Michoacán, Morelos, Hidalgo, Chihuahua, Zacatecas, Coahuila, Durango y Sonora (Jessup, 2002); aquí reportada para el municipio de Acultzingo, en donde es una planta común (Figs. 6D y 7). Especímenes examinados: México, Veracruz, Mpio. Acultzingo, cerro Xochío, paraje La Organera, A $1 \mathrm{~km}$ del poblado Sierra de Agua, alt. $1720 \mathrm{~m}$, 18²'34" N, 97¹4'54" W, 18 septiembre 2012, J. E. Rivera H. et al. 4938 (CORU, XAL); paraje Peña Roja, $1.2 \mathrm{~km}$ al O del poblado Próspero Pineda, alt. $1590 \mathrm{~m}$, 1844'42" N, 97²16'01" W, 21 julio 2014, J. E. Rivera H. et al. 6221 (CORU, MEXU, XAL).

\section{MALVACEAE}

Ceiba aesculifolia subsp. parvifolia (Rose) P.E. Gibbs et Semir. Árbol endémico de México, conocido de Guerrero, Morelos, Puebla y Oaxaca (Standley, 1920; Gibbs y Semir, 2003; Pagaza y Fernández, 2004). En el fascículo de Bombacaceae de la serie Flora de Veracruz (Avendaño, 1998), no se consideró a esta especie para el estado, sin embargo, ahora se colectó en Acultzingo, en donde es un habitante común, principalmente en las cañadas y partes bajas de los cerros (Figs. 3G y 7). Especímenes examinados: México, Veracruz, Mpio. Acultzingo, cerro Xochío, 1 km al NO de Tecamalucan, Alt. $1800 \mathrm{~m}, 10$ junio 2001, H. Oliva R. y M. J. Cházaro B. 3030 (CORU, MEXU, XAL); cerro Xochío, paraje La Organera, a $1 \mathrm{~km}$ al NO del poblado Sierra de Agua, Alt. 1720 m, 1845'34" N, 97¹4'54" W, 20 enero 2013, J. E. Rivera H. et al. 5452 (CORU, XAL); cerro Xochío, paraje la Organera, a $1 \mathrm{~km}$ al NO del poblado Sierra de Agua, Alt. 1720 m, 18²4'34" N, 97¹5'29" W, 22 marzo 2013, A. F. Vargas R. et al. 300 (CORU, MEXU, XAL); Paraje Peña Roja, $1.2 \mathrm{~km}$ al O del poblado Próspero Pineda, alt. $1590 \mathrm{~m}, 18^{\circ} 44^{\prime} 42^{\prime \prime} \mathrm{N}, 9^{\circ} 16^{\prime} 01^{\prime \prime} \mathrm{W}, 21$ julio 2014, J. E. Rivera H. et al. 6224 (CORU, XAL). 


\section{OPILIACEAE}

Agonandra racemosa (DC.) Standl. Conocida desde México hasta Guatemala, Honduras y El Salvador (Standley y Steyermark, 1946; Hiepko, 2000, 2008). En México se distribuye en la vertiente del Pacífico y centro-norte de México, en los estados de Sonora, Sinaloa, Chihuahua, Durango, Nayarit, Jalisco, Guanajuato, Colima, Estado de México, Michoacán, Morelos, Puebla, Zacatecas, Guerrero, Oaxaca y Chiapas (Carranza, 2000). Acevedo y Martínez (1995), en el fascículo de Opiliaceae de Veracruz, no consideraron a esta especie. Se le registra ahora de forma escasa en cañadas protegidas de los cerros del municipio de Acultzingo (Figs. 6C y 8). Especímenes examinados: México, Veracruz, Mpio. Acultzingo, cerro Xochío, 1 km al norte de Guadalupe, alt. 1419 m, 18²5'38.0" N, 97¹4'53.6" W, 27 mayo 2012, M. J. Cházaro B. et al. 10321 (XAL, XALU); cerro Xochío, paraje La Organera, a $1 \mathrm{~km}$ al NO del poblado Sierra de Agua, Alt. $1590 \mathrm{~m}, 1^{\circ} 45^{\prime} 33^{\prime \prime} \mathrm{N}, 97^{\circ} 14^{\prime} 37^{\prime \prime} \mathrm{W}, 25$ mayo 2013, J. E. Rivera H. et al. 5863 (CORU, MEXU, XAL); cerro Xochío, paraje La Organera, a $1 \mathrm{~km}$ al NO del poblado Sierra de Agua, alt. $1590 \mathrm{~m}, 18^{\circ} 45^{\prime} 33^{\prime \prime} \mathrm{N}$, 97²14'37" W, 5 octubre 2013, A. F. Vargas R. et al. 855 (CORU, MEXU, XAL).

\section{ORCHIDACEAE}

Dichromanthus cinnabarinus subsp. galeottianus (Schltr.) Soto Arenas et Salazar. Planta endémica de México, reportada como restringida en su distribución al Valle de Tehuacán-Cuicatlán, en los estados de Puebla y Oaxaca (Hágsater y Soto-Arenas, 2003). Aquí registrada del municipio de Acultzingo, donde es una especie común, aunque solo evidente en floración (Fig. 8). Especímenes examinados: México, Veracruz, Mpio. Acultzingo, cerro Xochío, paraje La Organera, a $1 \mathrm{~km}$ al NO del poblado Sierra de Agua, alt. 1590 m, 18²4'33" N, 97¹4'37" W, 5 octubre 2013, E. Rivera H. et al. 6101 (CORU, MEXU, XAL); cerro Xochío, paraje La Organera, a $1 \mathrm{~km}$ al NO del poblado Sierra de Agua, alt. 1720 m, 18²5'34" N, 97¹4'54" W, 28 junio 2014, A. F. Vargas R. et al. 1303 (CORU, MEXU, XAL).

\section{OROBANCHACEAE}

Silvia prostrata (Kunth) Benth. Planta herbácea endémica de México, reportada previamente para el Distrito Federal, Hidalgo, Estado de México, Oaxaca, Puebla y Tlaxcala (Méndez y Villaseñor, 2001). Se le encontró en las partes más altas del municipio de Aquila, en bosque de encino, en donde es habitante común (Figs. 3D y 8). Especíme- 


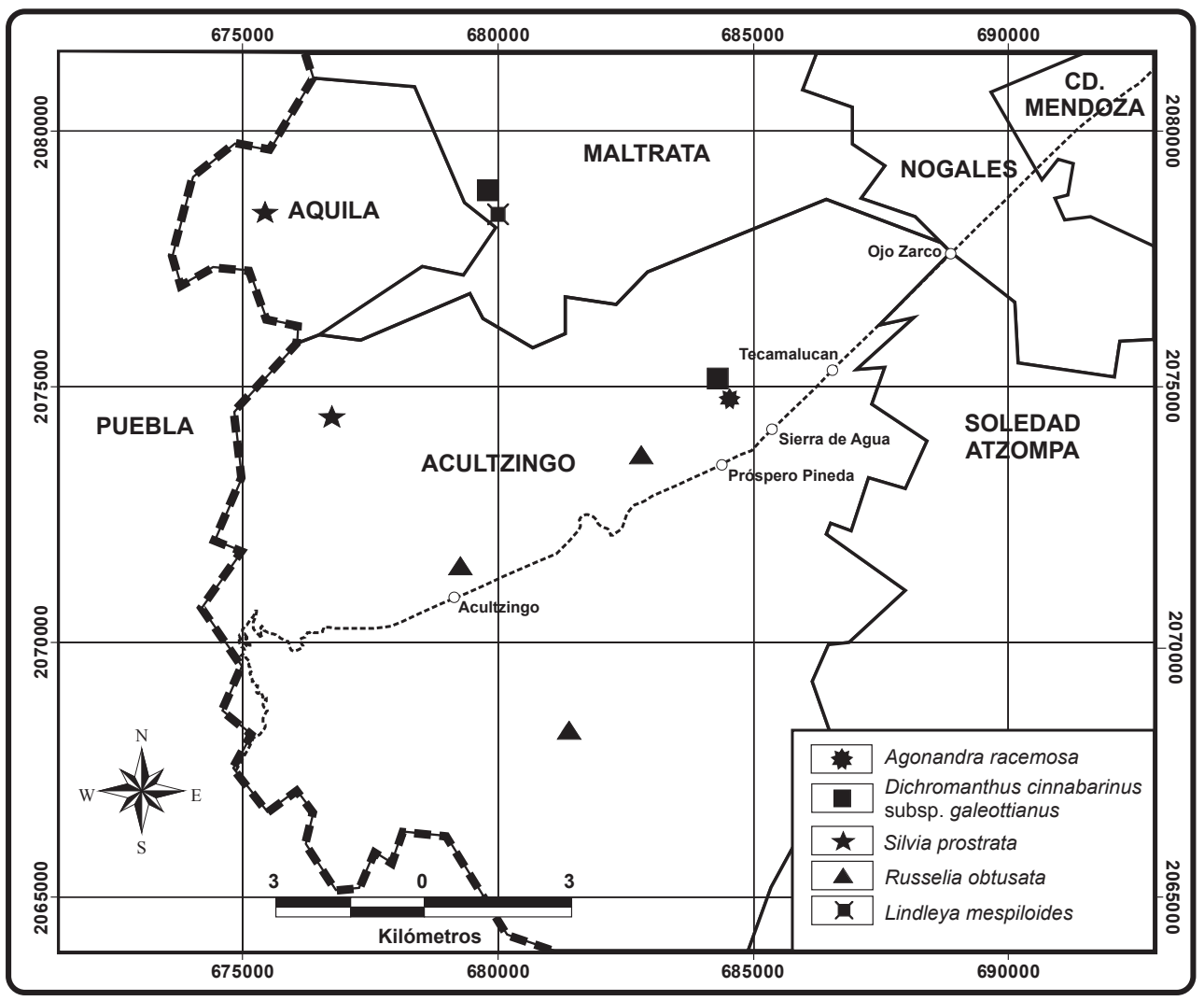

Fig. 8. Mapa de distribución en el área de estudio de Agonandra racemosa, Dichromanthus cinnabarinus subsp. galeottianus, Silvia prostrata, Russelia obtusata y Lindleya mespiloides.

nes examinados: México, Veracruz, Mpio. Aquila, camino a las antenas de microondas, a $2.5 \mathrm{~km}$ en línea recta al $\mathrm{O}$ de la localidad de Aquila, alt. $2480 \mathrm{~m}, 18^{\circ} 47^{\prime} 31^{\prime \prime} \mathrm{N}$, 97²0'10" W, 25 abril 2013, J. E. Rivera H. et al. 5717 (CORU, XAL). Mpio. Acultzingo, Ojo de Agua de Arriba, $5 \mathrm{~km}$ al NO en línea recta de la parada "El Mezquite", alt. 2370 m, 1845'16" N, 97¹9'22" W, 15 junio 2013, J. E. Rivera H. et al. 5937 (CORU).

\section{PLANTAGINACEAE}

Russelia obtusata S.F. Blake. Planta endémica de México, únicamente conocida de los estados de Puebla y Oaxaca (Méndez y Villaseñor, 2001). Se registra en el 
municipio de Acultzingo, donde es una especie común de las partes más bajas de los cerros (Figs. 3F y 8). Especímenes examinados: México, Veracruz, Mpio. Acultzingo, cerro por el camino al poblado El Potrero, aprox. $3 \mathrm{~km}$ en línea recta al SE de la parada "El Mezquite", alt. 1890 m, 18²'10" N, 97¹6'17" W, 1 septiembre 2012, J. E. Rivera H. y A. F. Vargas R. 4775 (CORU, XAL); cerro al norte de Acultzingo, por la ermita, alt. $1690 \mathrm{~m}, 18^{\circ} 43^{\prime} 41^{\prime \prime} \mathrm{N}, 97^{\circ} 17^{\prime} 50^{\prime \prime} \mathrm{W}, 16$ mayo 2014, A. F. Vargas $R$. et al. 1136 (CORU); paraje Peña Roja, $1.2 \mathrm{~km}$ al O del poblado Próspero Pineda, alt. $1590 \mathrm{~m}, 18^{\circ} 44^{\prime} 42^{\prime \prime} \mathrm{N}, 9^{\circ} 16^{\prime} 01^{\prime \prime} \mathrm{W}, 21$ julio 2014, J. E. Rivera H. et al. 6214 (CORU, MEXU, XAL).

\section{ROSACEAE}

Lindleya mespiloides Kunth. Árbol endémico de México, reportado de Chihuahua, Coahuila, Nuevo León, Tamaulipas, Durango, Zacatecas, Aguascalientes, San Luis Potosí, Querétaro, Hidalgo, Puebla y Oaxaca (Rzedowski, 2005); ahora se amplía su distribución para Veracruz, del municipio de Maltrata, en las partes altas de los cerros, en donde es escaso (Figs. 6A y 8). Espécimen examinado: México, Veracruz, Mpio. Maltrata, cerro al SO de Maltrata, a $1.5 \mathrm{~km}$ en línea recta del centro del pueblo, alt. 2100 m, 1847'31" N, 97²1'36" W, 17 mayo 2014, A. F. Vargas R. et al. 1172 (CORU, MEXU, XAL).

\section{DISCUSIÓN Y CONCLUSIONES}

De las 19 especies aquí tratadas, 14 son de distribución restringida de México y seis estaban consideradas como endémicas del Valle de Tehuacán-Cuicatlán; 17 de todas ellas están presentes en el mencionado Valle y otras dos (T. galanthoides y $S$. prostrata, del bosque de encino), se registran también de diferentes localidades de Oaxaca y/o Puebla. De las 19, se reportan tres para el municipio de Maltrata, dos para Maltrata y Acultzingo y 14 solo para el de Acultzingo. El mayor número de especies encontradas en Acultzingo se debe, muy probablemente, a que se realizó más esfuerzo de colecta en ese municipio; sin embargo, también se observó allí una mayor variación en lo referente a humedad, orientación de la ladera y otros factores.

Por otra parte, los municipios de Acultzingo y Maltrata, por su ubicación geográfica en el centro de Veracruz y en la frontera con el estado de Puebla, comparten elementos con la flora del estado de Puebla. Tal y como se discute en Rivera-Her- 
nández et al. (2014), la provincia florística del Valle de Tehuacán-Cuicatlán incluye, según Rzedowski (1978: 107), un área relativamente reducida del sureste del estado de Puebla y porciones adyacentes del de Oaxaca, abarcando también una pequeña superficie del estado de Veracruz, que probablemente se refería a los municipios de Acultzingo y Maltrata, por lo que de este modo se puede discernir que tales dos municipios veracruzanos forman parte de la Provincia Florística de TehuacánCuicatlán.

Esta afirmación se refuerza con el registro de 17 de las 19 especies reportadas en este artículo que se comparten con el Valle de Tehuacán-Cuicatlán, con los ocho nuevos registros de cactáceas para Acultzingo proporcionados por RiveraHernández et al. (2014) y con otros elementos compartidos entre estas dos regiones, tales como: Gochnatia obtusata S. F. Blake, Quercus sebifera Trel., Rhus standleyi F. A. Barkley, Pinguicula moranensis Kunth, Ipomoea conzattii Greenm., Bursera fagaroides (Kunth) Engl., Tecoma stans (L.) Juss. ex Kunth y Gymnosperma glutinosum (Spreng.) Less., entre varias más. Con toda esta evidencia, se confirma que los municipios aquí estudiados forman parte del Valle de Tehuacán-Cuicatlán, por lo que deben ser incluidos en futuras investigaciones taxonómicas y biogeográficas como parte del mismo.

Finalmente, el estudio botánico y fitogeográfico de esta región semiárida del centro de Veracruz es prioritario, tanto para conocer mejor su composición florística y relaciones fitogeográficas, como para poder establecer, de una mejor manera, estrategias de conservación y manejo. Tomando en cuenta que esta región ha permanecido olvidada, son necesarias más exploraciones botánicas y una pesquisa más intensiva.

\section{AGRADECIMIENTOS}

Agradecemos a los diferentes especialistas por la identificación o confirmación de las diferentes taxones aquí tratados: Raúl Acevedo (Opiliaceae), Jaime Jiménez y Martha Martínez (Euphorbiaceae), Abisaí García-Mendoza (Asparagaceae e Iridaceae), Gerardo Salazar (Orchidaceae), Rosalinda Medina-Lemos (Burseraceae y Araliaceae) y Steve L. Jessup (Malpighiaceae). Nuestro agradecimiento también a Burl L. Mostul, Brian Kamble, Kelly Griffins, Alberto Badía, Lorenzo Escandón, Gerardo Torres, David Jimeno, Rebeca Varo, Juan Antonio Francisco, José Luis Pacheco, Sergio Morales-Juan y Oscar Cid por su ayuda y compañía durante el trabajo de campo. A Gonzalo Castillo-Campos por la revisión crítica 
del manuscrito final que enriqueció sustancialmente el mismo. Se agradece a dos revisores anónimos por sus útiles sugerencias para mejorar el artículo.

\section{LITERATURA CITADA}

Acevedo R., R. y J. L. Martínez y P. 1995. Opiliaceae. Flora de Veracruz 84: 1-6.

Angiosperm Phylogeny Group. 2009. An update of the Angiosperm Phylogeny Group classification for the orders and families of flowering plants: APG III. Bot. J. Linn. Soc. 161: 105-121.

Anónimo. 2010. Mapa de los municipios de México. Marco Geoestadístico Nacional versión 5.0.A. Censo de Población y Vivienda 2010. Instituto Nacional de Estadística y Geografía. Aguascalientes, México.

Avendaño R., S. 1998. Bombacaceae. Flora de Veracruz 107: 1-140.

Carranza G., E. 2000. Opiliaceae. Flora del Bajío y de Regiones Adyacentes 81: 1-7.

Castillo-Campos, G, S. Avendaño R. y M. E. Medina A. 2011. Flora y vegetación. La biodiversidad en Veracruz: Estudio de estado. Volumen I. Comisión Nacional para el Conocimiento y Uso de la Biodiversidad (CONABIO), Gobierno del Estado de Veracruz, Universidad Veracruzana e Instituto de Ecología, A.C. México, D.F., México. pp. 163-179.

Diggs, G. M. 1995. Ericaceae. Part II. The superior-ovaried genera (Monotropoideae, Pyroloideae, Rhododendroideae, and Vaccinioideae). Flora Neotropica Monograph 66: 146-193.

Dressler, R. L. 1961. A synopsis of Poinsettia (Euphorbiaceae). Ann. Missouri Bot. Gard. 48: 329-341.

Espejo S., A. y A. R. López-Ferrari. 1998. Iridaceae. Flora de Veracruz 105: 1-58.

García-Mendoza, A. J. 2011. Agavaceae. Flora del Valle de Tehuacán-Cuicatlán 88: 1-95 pp.

Gibbs, P. y J. Semir. 2003. Revisión taxonómica del género Ceiba Mill. (Bombacaceae). Anales Jard. Bot. Madrid 60(2): 259-300.

Gómez-Pompa, A. 1977. Ecología de la vegetación del estado de Veracruz. Instituto de Investigaciones sobre Recursos Bióticos, A.C. y CECSA. Xalapa, México. 91 pp.

Gómez-Pompa, A., T. Krömer y R. Castro-Cortés (coords.) 2010. Atlas de la flora de Veracruz. Un patrimonio natural en peligro. Gobierno del Estado de Veracruz y Universidad Veracruzana. Xalapa, México. 528 pp.

Hágsater, E. y M. A. Soto-Arenas. 2003. Orchids of Mexico. Icon. Orchid. 5-6: i-xxii, t. 501-t. 700.

Hiepko, P. 2000. Opiliaceae. Flora Neotropica Monograph 82: 1-55.

Hiepko, P. 2008. Opiliaceae. Species Plantarum: Flora of the World 12: 1-71.

Jessup, S. L. 2002. Six new species and taxonomic revisions in Mexican Gaudichaudia (Malpighiaceae). Madroño 49(4): 237-255.

López-Ferrari, A. R. 1993. Araliaceae. Flora del Bajío y de Regiones Adyacentes 20: 1-16 pp. 
Lot, A. y F. Chiang (comps.). 1986. Manual de herbario. Consejo Nacional de la Flora de México, A.C. México, D.F., México. 142 pp.

Medina-Lemos, R. 1994. Araliaceae. Flora del Valle Tehuacán-Cuicatlán 4: 1-13.

Medina-Lemos, R. 2008. Burseraceae. Flora del Valle Tehuacán-Cuicatlán 66: 1-46.

Méndez L., I. y J. L. Villaseñor R. 2001. La familia Scrophulariaceae en México: diversidad y distribución. Bol. Soc. Bot. Méx. 69: 101-121.

Mostul, B. L. y M. Cházaro B. 1996. Two geophytic Euphorbias from western Mexico. Cact. Succ. J. 68(3): 153-155.

Pagaza C., E. y R. Fernández N. 2004. La familia Bombacaceae en la cuenca del Río Balsas, México. Polibotánica 17: 71-102.

Ramos A., C. H. y F. González M. 1972. La vegetación de la zona árida veracruzana. Anales Inst. Biol. Univ. Nac. México. Ser. Bot. 43(1): 77-99.

Rivera-Hernández., J. E., J. Reyes Santiago, M. Cházaro Basañez., F. Ramón, A. F. Vargas y G. Alcántara. 2014. Las cactáceas del municipio de Acultzingo, Veracruz. Cact. Suc. Mex. 59(3): 68-78.

Rivera L., M. y E. Solano. 2012. Nolinaceae. Flora del Valle de Tehuacán-Cuicatlán 99: 1-26.

Rodríguez A., M., K. Vega F., V. H. De Gante C. y J. Jiménez R. 2009. Distribución del género Jatropha L. (Euphorbiaceae) en el estado de Puebla, México. Polibotánica 28: 37-48.

Rzedowski, J. 1978. La vegetación de México. Editorial Limusa. México, D.F., México. 432 pp.

Rzedowski, J. y G. Calderón de R. 1987. Nota sobre Euphorbia stormiae (Euphorbiaceae). Cact. Suc. Mex. 32(4): 75-77.

Rzedowski, J. 1991. El endemismo en la flora fanerogámica mexicana: una apreciación analítica preliminar. Acta Bot. Mex. 15: 47-64.

Rzedowski, J. y F. Guevara-Fefer. 1992. Burseraceae. Flora del Bajío y de Regiones Adyacentes 3: 1-46.

Rzedowski, J. y G. Calderón de R. 1996. Burseraceae. Flora de Veracruz 94: 1-37.

Rzedowski, G. C. de, J. Rzedowski y colaboradores. 2001. Flora fanerogámica del Valle de México. 2a ed. Instituto de Ecología, A.C. y Comisión Nacional para el Conocimiento y Uso de la Biodiversidad. Pátzcuaro, México. 1406 pp.

Rzedowski, J. 2005. Rosaceae. Flora del Bajío y de Regiones Adyacentes 135: 1-157.

Sandoval J., M. S. 1984. Estudio de la vegetación de la Sierra de Mastaloyan, PueblaVeracruz. Tesis de licenciatura. Universidad Veracruzana. Xalapa, México. 78 pp.

Sosa, V. 1979. Araliaceae. Flora de Veracruz 8: 1-38.

Standley, P. C. 1920. Trees and shrubs of Mexico. Contr. U.S. Natl. Herb. 23(1): 1-791.

Standley, P. C. y J. A. Steyermark. 1946. Opiliaceae. Flora of Guatemala. Field. Bot. 24(4): 86-87. 